\title{
An Experimental Study and a Numerical Simulation of the Turbulent Flow under the Vortex Finder of a Cyclone Separator
}

\author{
K. Talbi ${ }^{1}$, Z. Nemouchi ${ }^{1}$, A. Donnot ${ }^{2}$ and N. Belghar ${ }^{1}$ \\ ${ }^{1}$ LEAP, Département de Génie Mécanique, Université Mentouri Constantine \\ Route de Ain-El-Bey, Constantine, 25000, Algeria \\ ${ }^{2}$ LERMAB UMR/INRA 1093, Université Henry Poincaré Nancy \\ BP 239, 54506 Vandoeuvre Cedex France \\ Email:talbi.kamel1@gmail.com
}

(Received January 5, 2009; accepted October 14, 2009)

\begin{abstract}
An experimental study and a numerical simulation are presented concerning the three dimensional turbulent flow of air in a cyclone separator in the region underneath the vortex finder. The computations are carried out using the Fluent CFD code. The turbulence effects on the mean flow are taken into account using the standard k- $\varepsilon$ model and the standard Reynolds stress Model (RSM). The axial and tangential mean velocity components and the turbulence intensities are measured using Laser Doppler Anemometry. The LDA system is mounted in such a way that radial traverses at different angles of the cyclone cylindrical geometry and at different axial positions could be possible. The obtained results show interesting phenomena such as the three dimensional nature of the flow behaviour, the turbulence decay and its evolution towards an isotropic state in the quasi-free vortex region as the flow proceeds downstream in the cyclone. In the region underneath the vortex finder, the surface separating the descending and the ascending streams (set of points where the axial velocity component is nil) is located approximately in the fictitious prolongation of the vortex finder cylindrical wall. The existence of a quasi-forced vortex in the central region of the cyclone surrounded by a coaxial quasi-free vortex is confirmed. The radial distance separating the central vortex and the surrounding annular vortex, at a given angle and axial position, can be clearly defined as the distance from the axis to the point of intersection between two characteristic straight lines: the first line representing, $\ln U t \mathrm{vs} \ln \mathrm{r}$, of slope +1 , in the quasi-forced vortex and the second line, ln Ut vs $\ln r$, of slope -1 , in the quasi-free vortex, where Ut is the tangential component of the mean velocity.
\end{abstract}

Keywords: Cyclone separator, free vortex, forced vortex, Laser Doppler Anemometry, k- $\varepsilon$ model, Reynolds stress model, Finite-volume method.

\section{NOMENCLATURE}

\begin{tabular}{|c|c|c|c|}
\hline$D_{i, j}$ & diffusion rate of $u_{i} u_{i}$ & $\varepsilon$ & dissipation rate of $k$ \\
\hline$k$ & turbulent kinetic energy & $\varepsilon_{i, j}$ & dissipation rate of $u_{i} u_{j}$ \\
\hline$n_{i}$ & $\begin{array}{l}\mathrm{x}_{\mathrm{i}} \text { component of the unit vector } \\
\text { normal to the wall }\end{array}$ & $\theta$ & tangential coordinate \\
\hline$P_{i}$ & production rate of $\overline{u_{i} u_{j}}$ & $v, v_{t}$ & $\begin{array}{l}\text { laminar and turbulent kinematic } \\
\text { viscosities }\end{array}$ \\
\hline$\overline{U_{i}}$ & $\begin{array}{l}\mathrm{x}_{\mathrm{i}} \text { component of the mean } \\
\text { velocity }\end{array}$ & $\sigma_{k, L}, \sigma_{k, t}$ & $\begin{array}{l}\text { laminar and turbulent Prandtl } \\
\text { numbers associated with } k\end{array}$ \\
\hline Ut & $\begin{array}{l}\text { tangential component of the } \\
\text { mean velocity }\end{array}$ & $\sigma_{\varepsilon, L}, \sigma_{\varepsilon, t}$ & $\begin{array}{l}\text { laminar and turbulent Prandtl } \\
\text { numbers associated with } \varepsilon\end{array}$ \\
\hline$u_{i} u_{j}$ & the distance normal to the wall & $\phi_{i j, 1}, \phi_{i j, 2}, \phi_{i j, w}$ & pressure-strain correlation terms \\
\hline
\end{tabular}




\section{INTRODUCTION}

The cyclone separator is a simple and practical device used in industry, to separate gaseous, liquid and/or solid phases. The mixture enters with a tangential velocity that is sufficiently high for an efficient separation of the phases to occur. The heavy phase is transportedtowards the outer wall because of the centrifugal acceleration. The study of the complex phenomena characterizing the three dimensional turbulent multi-phase flow within the cyclone is a challenge for the researchers motivated by the need to improve the cyclone separation efficiency and those concerned with the establishment of mathematical models describing satisfactorily the existing phenomenology.

Previous works concerning the aerodynamics within a cyclone have been reported in the literature based on experiments (e.g. Ogawa 1981 using hot wire anemometry, Monredon et al. 1992; Fraser and Abdullah 1995; Potel et al. 1997 and Obermair et al. 2001; Peng et al. 2001 using Laser Doppler Anemometry), based on numerical methods assuming axisymmetry (e.g. Boysan et al. 1982; Slack et al. 2003; Ayers et al. 1983 and Peng et al. 2001) or considering three dimensional flows (e.g. Frank et al. 1998; Frank et al. 1999; Hishoda 1996; Matvienko et al. 2001; Derksen and Van den Akker 2002; Zhou and Soo 1990; Brennan 2003 and Raoufi et al. 2007).

In view of the lack of experimental data concerning the mean and turbulent characteristics of the $3 \mathrm{D}$ flow in question, the work reported in this paper is an experimental and a numerical study of the aerodynamics in a cyclone separator. In the first part of the work the mean velocity components and the turbulent intensities in the axial and tangential directions are measured by the LDA system. In the second part, the $3 \mathrm{D}$ flow is computed numerically using the standard k- $\varepsilon$ turbulence model and the RSM. The region underneath the vortex finder is considered in particular where the prediction of the characteristic features is a real challenge, namely, the behaviour of the quasi-forced and quasi-free vortices, and the opposing ascending and descending streams. It is hoped that the results presented hereunder can be seen as a contribution to the flow analysis of the existing complex phenomena on the one hand and a set of data to validate $3 \mathrm{D}$ CFD codes and to test the performance of turbulence models on the other.

\section{EXPERIMENTAL RIG}

The experimental rig, Fig. 1, consists of three essential parts: the LDA system, the cyclone and the displacement system of the optical probe. The 3D computer-controlled displacement system was conceived so that the velocity components in the tangential and axial directions could be measured in various parts of the cyclone domain. The optical probe could be displaced in the radial, tangential and axial directions. Radial profiles of the axial and the tangential velocity components were measured at the axial positions $\mathrm{z}=765 \mathrm{~mm}$ and $\mathrm{z}=855 \mathrm{~mm}$ underneath the vortex finder, Fig. 2, and at different angles, from $\theta=$ $10^{\circ}$ to $\theta=230^{\circ}$ by steps of $20^{\circ}$, Fig. 3 .

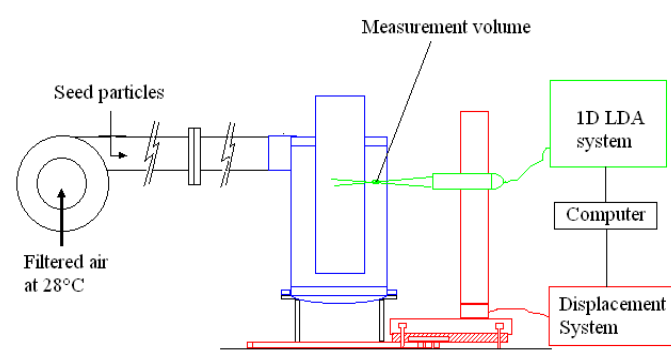

Fig. 1. Experimental rig.

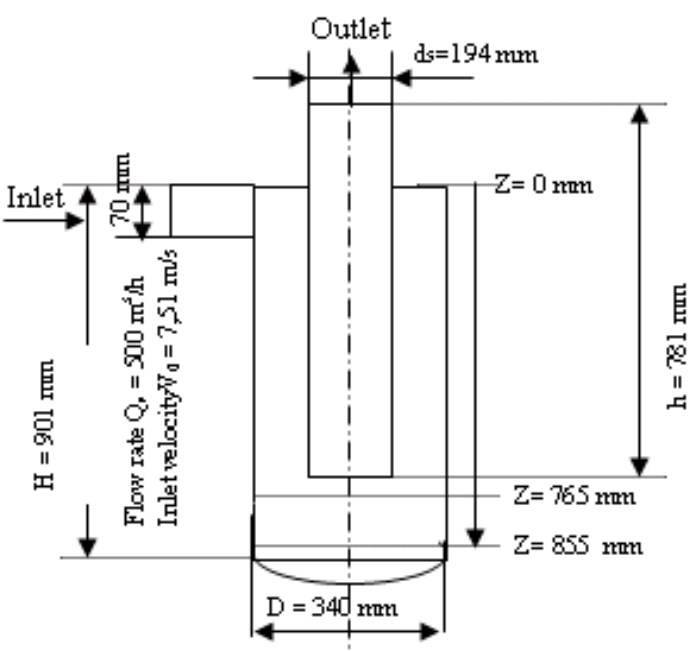

Fig. 2. Axial measuring positions under the vortex finder.

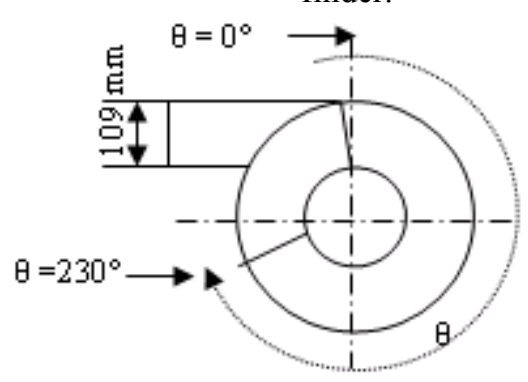

Fig. 3. Angular measuring positions: $-10^{\circ}<\theta<230^{\circ}$ by steps of $20^{\circ}$.

A one-component back-scatter LDA system is used, consisting of a $5 \mathrm{~W}$ argon ion laser, a beam splitter, a brag cell, a lens with a focal length of $350 \mathrm{~mm}$, a photodetector and an IFA750 signal processor. The seed particles, backscattering the light back into the detector, are injected as shown in Fig. 1.

At each point, 5000 measurements were performed at an acquisition rate of $4 \mathrm{kHz}$.

\section{TurbulenCE Modelling}

Two models of turbulence are used in the present work, namely, the standard k- $\varepsilon$ model and the standard RSM.

\subsection{The Standard k- $\varepsilon$}


The semi-empirical form of the turbulent kinetic energy equation used is:

$$
\begin{aligned}
& \frac{\partial k}{\partial t}+\overline{U_{j}} \frac{\partial k}{\partial x_{j}}=v_{t}\left(\frac{\partial \overline{U_{i}}}{\partial x_{j}}+\frac{\partial \overline{U_{j}}}{\partial x_{i}}\right) \frac{\partial \overline{U_{i}}}{\partial x_{j}}+ \\
& \stackrel{1}{\longleftrightarrow} \stackrel{\longleftrightarrow}{\longleftrightarrow} \stackrel{\frac{\partial}{\partial x_{j}}\left(\left(\frac{v}{\sigma_{k, L}}+\frac{v_{t}}{\sigma_{k, t}}\right) \frac{\partial k}{\partial x_{j}}\right)-\varepsilon}{\longrightarrow}
\end{aligned}
$$

- Term 1 is the rate of change of the turbulent kinetic energy along a mean streamline.

- Term 2 is the rate of production of $\mathrm{k}$.

- Term 3 is the transport of $\mathrm{k}$ by molecular interaction and by the turbulence.

- $\quad$ Term 4 is the rate of dissipation of $k$.

The modelled form of the dissipation rate equation is:

$$
\begin{aligned}
& \frac{\partial \varepsilon}{\partial t}+\bar{U} j \frac{\partial \varepsilon}{\partial x_{j}}=C_{\varepsilon 1} \frac{\varepsilon}{k} v_{t}\left(\frac{\partial \overline{U_{i}}}{\partial x_{j}}+\frac{\partial \overline{U_{i}}}{\partial x_{j}}\right) \frac{\partial \overline{U_{i}}}{\partial x_{j}}+ \\
& \stackrel{{ }_{a}}{\longleftrightarrow} \stackrel{\longleftrightarrow}{\longleftrightarrow x_{j}\left[\left(\frac{v}{\sigma_{\varepsilon, L}}+\frac{v_{t}}{\sigma_{\varepsilon, t}}\right) \frac{\partial \varepsilon}{\partial x_{j}}\right]-C_{\varepsilon 2} \frac{\varepsilon^{2}}{k}} \stackrel{{ }_{d}}{\longleftrightarrow}
\end{aligned}
$$

- Term a is the rate of change of $\varepsilon$ along a mean streamline.

- $\quad$ Term $b$ is the rate of production of $\varepsilon$.

- Term $\mathrm{c}$ is the transport of $\varepsilon$ by molecular interaction and by the turbulence.

- $\quad$ Term $\mathrm{d}$ is the rate of destruction of $\boldsymbol{\varepsilon}$.

The diffusive terms are of the gradient type. $\sigma_{k, L}$ and $\sigma_{k, t}$ are the laminar and turbulent Prandtl numbers associated with k respectively. $\sigma_{\varepsilon, L}$ and $\sigma_{\varepsilon, t}$ are the laminar and turbulent Prandtl numbers associated with $\varepsilon$.The obtained values of $\mathrm{k}$ and $\varepsilon$ from Eqs. (1) and (2) give the turbulent viscosity which in turn yields the Reynolds stresses using the Boussinesq eddy viscosity concept.

\subsection{The Standard RSM}

The $\overline{u_{i} u_{j}}$ Reynolds stress transport equation can be written:

$$
\begin{aligned}
& \frac{D \overline{\left(u_{i} u_{j}\right)}}{D t}=P_{i, j}+D_{i, j}-\varepsilon_{i, j} \\
& +\phi_{i j, 1}+\phi_{i j, 2}+\phi_{i j, w}
\end{aligned}
$$

Where $P_{i, j}$ is the production rate of $\overline{u_{i} u_{j}}, D_{i, j}$ the diffusion rate, $\varepsilon_{i, j}$ the dissipation rate and $\phi_{i j, 1}, \phi_{i j, 2}$ ,$\phi_{i j, w}$ are the three contributions to the pressure-strain correlation considered below.

The production term $P_{i, j}$ is not modelled as it involves mean velocities and Reynolds stress components which are among the principal unknowns in the system of equations. It is given by:

$$
P_{i, j}=-\overline{u_{i} u_{k}} \frac{\partial \overline{U_{j}}}{\partial x_{k}}-\overline{u_{j} u_{k}} \frac{\partial \overline{U_{i}}}{\partial x_{k}}
$$

The rate of destruction term $\varepsilon_{i, j}$ is assumed to be equal to $\frac{2}{3} \varepsilon \delta_{i, j}$, where $\varepsilon$ is the rate of dissipation of the turbulent kinetic energy. The fluid viscosity dissipates most of the turbulent energy through the smallest eddies (the Kolmogorv scale) which behave isotropically on average. Thus, approximately, the rates of destruction of the normal stresses $\overline{u_{i}{ }^{2}}$ will be taken as equal and those of the tangential stresses $\overline{u_{i} u_{j}}$ (with $\mathrm{i} \neq \mathrm{j}$ ), considered as nil.

The pressure-strain correlation term comprises three parts. It represents an exchange or a redistribution between the stress components without effecting the sum of the normal stresses ie without effecting the turbulent energy, Rodi (1980).

The first part, representing a return to isotropy by purely turbulent interactions, is modelled as, Rotta (1961):

$$
\phi_{i j, 1}=-C_{1} \frac{\varepsilon}{k}\left(\overline{u_{i} u_{j}}-\frac{2}{3} \delta_{i j} k\right)
$$

, where $\mathrm{C}_{1}=1.5$.

It is proportional to the turbulence anisotropy, with an opposite sign. In fact, in the $\overline{u_{i}^{2}}$ equation, it is a source if $\overline{u_{i}{ }^{2}}<\frac{2}{3} k$ and a sink if $\overline{u_{i}{ }^{2}}>\frac{2}{3} k$ whence the turbulent energy redistribution between the normal stresses.

The second part, taking account of the return to isotropy by interaction between the turbulence and the mean velocity gradients is taken as, Launder et al. (1975):

$\phi_{i j, 2}=-\gamma\left(P_{i, j}-\frac{2}{3} \delta_{i j} P\right)$

where $\gamma=0.6, \quad P_{i j}=-\overline{u_{i} u_{k}} \frac{\partial \overline{U_{j}}}{\partial x_{k}}-\overline{u_{j} u_{k}} \frac{\partial \overline{U_{i}}}{\partial x_{k}} \quad$ and $P=-\overline{u_{i} u_{j}} \frac{\partial \overline{U_{i}}}{\partial x_{j}}$.

The third part, the wall reflection term, is responsible for the damping of the velocity fluctuation component close, and perpendicular, to the wall. It is modelled as follows: 


$$
\begin{gathered}
\phi_{i j, w} \equiv C^{\prime} \frac{\varepsilon}{k}\left(\overline{u_{k} u_{m}} n_{k} n_{m} \delta_{i j}-\frac{3}{2} \overline{u_{i} u_{j}} n_{j} n_{k}-\frac{3}{2} \overline{u_{j} u_{k}} n_{i} n_{k}\right) \frac{C_{l} k^{3 / 2}}{\varepsilon y_{p}} \\
+C_{2}^{\prime}{ }_{2}\left(\phi_{k m, 2} n_{k} n_{m} \delta_{i j}-\frac{3}{2} \phi_{i j, 2} n_{j} n_{k}-\frac{3}{2} \phi_{i k, 2} n_{i} n_{k}\right) \frac{C_{l} k^{3 / 2}}{\varepsilon y_{p}}
\end{gathered}
$$

where $\mathrm{C}_{1}=0.5, \mathrm{C}_{2}{ }_{2}=0.3, \mathrm{n}_{\mathrm{k}}$ is the $\mathrm{x}_{\mathrm{k}}$ component of the unit vector normal to the wall, $\mathrm{y}_{\mathrm{p}}$ the distance normal to the wall

$C_{l}=C_{\mu}^{3 / 4} / \kappa$ where $C_{\mu}=0.09$ and the Von Karman constant $\kappa=0.4187$.

The dominant diffusion term in Eq. (3) is that by the turbulent fluctuations, i.e.:

$$
D_{i, j}=\frac{\partial}{\partial x_{k}}\left(-\overline{u_{i} u_{j} u_{k}}\right)
$$

It is modelled as, Lien and Leschziner (1994):

$$
D_{T, i j}=\frac{\partial}{\partial x_{k}}\left(\frac{\mu_{t}}{\sigma_{k}} \frac{\partial \overline{u_{i} u_{j}}}{\partial x_{k}}\right)
$$

$$
\sigma_{\mathrm{k}}=0.82
$$

The Fluent code, based on the finite volume method, is used to simulate the flow in question.. The numerical mesh generated by the Gambit code, is illustrated in Fig. 4. The standard wall function is used for the nearwall treatment. The QUICK scheme is used to discretize the convective term in the transport equations.

\section{RESUlts AND DiscuSSION}

\subsection{Mean Velocities}

Figure 5a shows the profiles of the tangential component of the mean velocity at the axial position $\mathrm{z}=$ $765 \mathrm{~mm}$ underneath the vortex finder, for different angles. Experimental profiles obtained by the LDA system at the angles $\theta=0,110,180,230,290^{\circ}$ are illustrated together with the numerical profiles at the angles $\theta=90,270^{\circ}$ using both the k- $\varepsilon$ model and RSM. A significant discrepancy is observed between the computed results using the $\mathrm{k}-\varepsilon$ model and the experimental data. The tangential velocity is seriously underestimated and the location of the surface separating the free vortex from the forced vortex is not well predicted. The RSM seems to perform better in capturing the turbulence effects on the mean flow. Comparing the profiles of the axial component of the mean velocity at $\mathrm{z}=765 \mathrm{~mm}$ and $\mathrm{z}=855 \mathrm{~mm}$ and at $\theta=$ $0^{\circ}, 50^{\circ}, 110^{\circ}, 180^{\circ}, 230^{\circ}$ et $290^{\circ}$, Fig. $5 \mathrm{~b}$, the damping of the axial velocity magnitude is clearly observed as the bottom wall of the cyclone is approached. On the other hand, the profile of the tangential component remains practically unchanged: the maximum value at $\mathrm{z}=765 \mathrm{~mm}$ and $\mathrm{z}=855 \mathrm{~mm}$ is about $19 \mathrm{~m} / \mathrm{s}$. The fictitious cylindrical surface composed of the set of points where the axial velocity is zero, that is the surface which separates the descending fluid coming from the annular space and the ascending
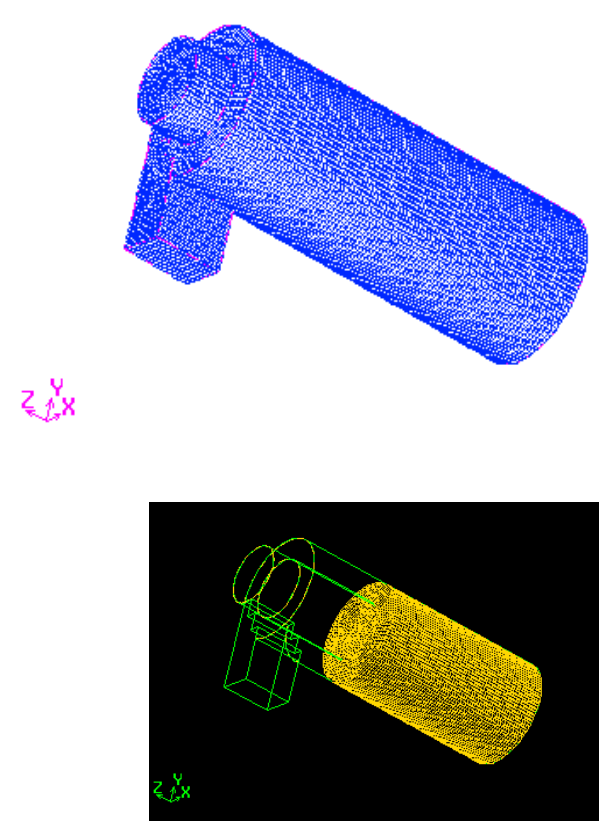

Fig. 4. Numerical mesh.

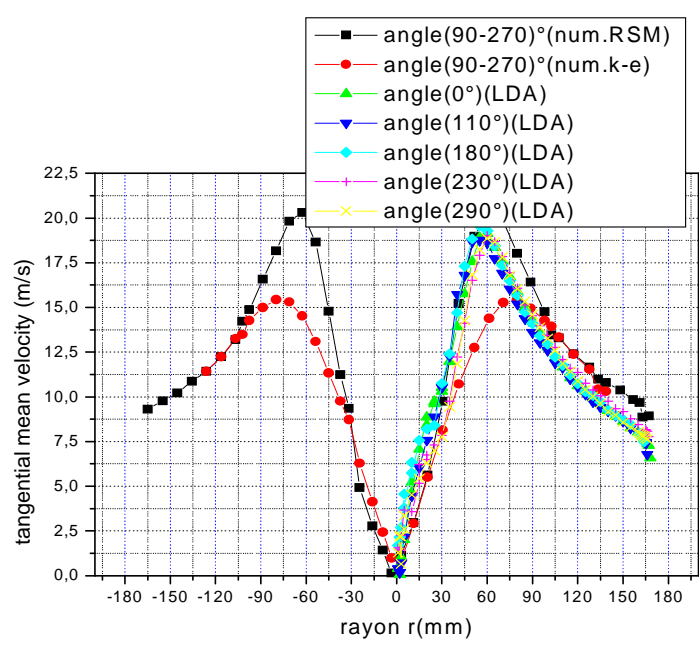

a) Tangential component

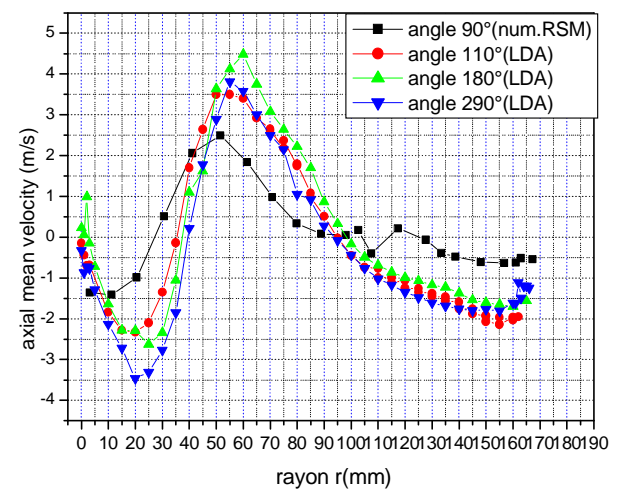

b) Axial component

Fig. 5. Mean velocity profiles at $\mathrm{z}=765 \mathrm{~mm}$. 
fluid moving towards the vortex finder annular, is located approximately on the prolongation of the vortex finder wall, Fig. 6a. Concerning the tangential velocity field under the exit tube, another fictitious surface is noted separating a central zone turning like a quasi forced vortex and an annular zone behaving like a quasi free vortex, Figs. $6 \mathrm{a}$ and $6 \mathrm{~b}$. To locate the separating surface, it could be defined as the set of points where two straight lines intersect. The first one representing approximately the variation of $\ln \left(\mathrm{U}_{\mathrm{t}}\right)$ as a function of $\ln (r)$ with a slope of +1 in the quasi-forced vortex region; the second one the variation of $\ln \left(\mathrm{U}_{\mathrm{t}}\right)$ as a function of $\ln (r)$ with a slope of -1 in the quasi-free vortex region. This is illustrated in Fig. 6c showing, as an example, the point separating the two types of vortex at $\mathrm{z}=765 \mathrm{~mm}$ and $\theta=0^{\circ}$. This result confirms Ogawa's proposal , Ogawa (1981) who considered the superposition of the two types of vortex. It should also be noted the velocity components are not in general equal to zero on the axis of the cyclone, Figs. 5 and 6.

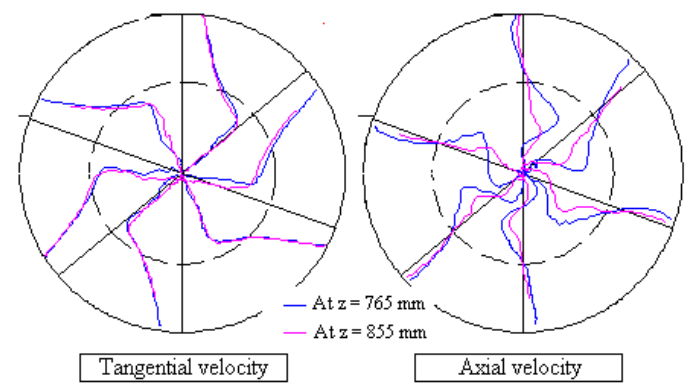

Fig. 6a. Tangential mean velocity profiles at different angles.

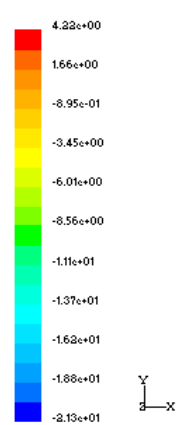

Separation zone between forced and free vortex

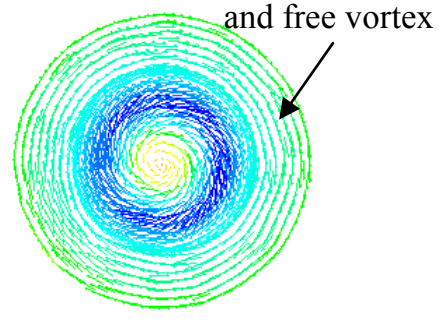

Fig. 6b. Projected velocity vectors at $\mathrm{z}=765 \mathrm{~mm}$, (units of colored scale in $\mathrm{m} / \mathrm{s}$ ).

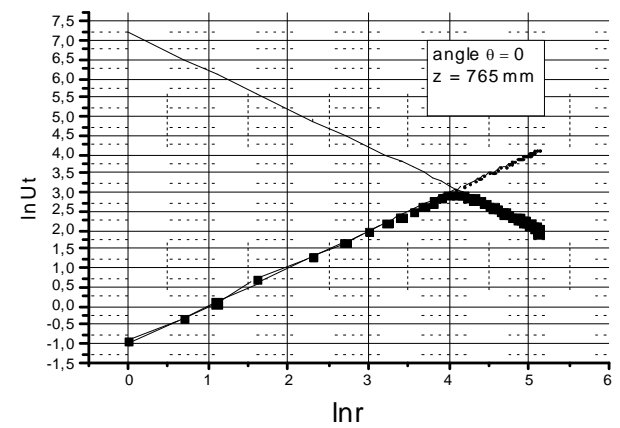

Fig. 6c. Mean tangential velocity profile in quasiforced vortex and quasi-free vortex regions.
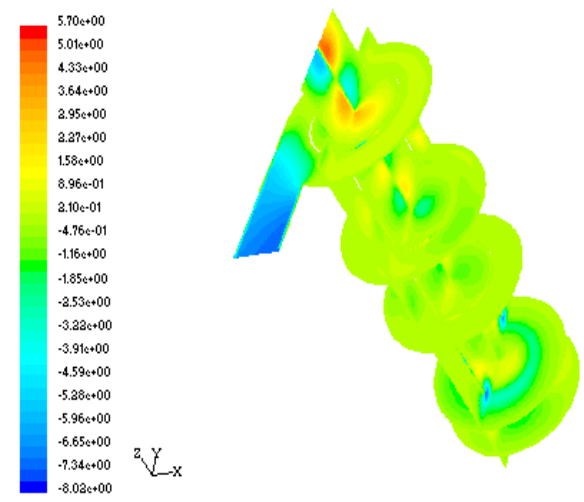

a) Radial component
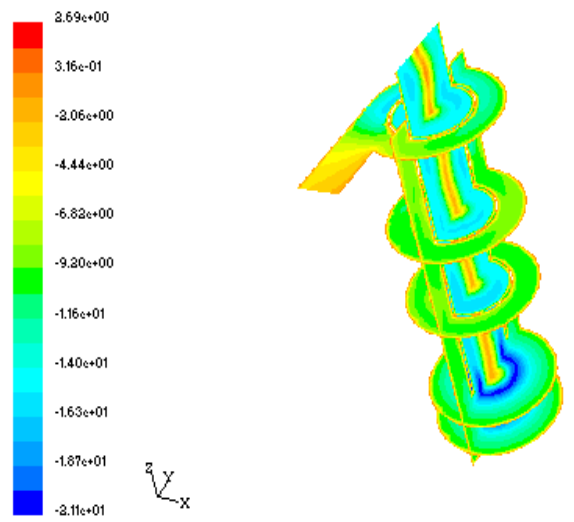

b) Tangential component

Fig. 7. Contours of mean velocity components (m/s) using the RSM.

Referring to Fig. 7 displaying contours of the radial and tangential components of the mean velocity, it can be seen that the centre of the swirling flow oscillates around the geometry axis. This phenomenon has been reported previously, see e.g. Derksen and Van den Akker (2002) who studied a cylindrical cyclone similar in shape to the one considered in this work. Negative values of the axial component of the mean velocity are observed at $\mathrm{z}=765 \mathrm{~mm}$ for the angles $\theta=180^{\circ}, 230^{\circ}$ and $290^{\circ}$ near the cyclone axis. A small stream is, in fact, flowing downward within a sector in the central region under the vortex finder. The depression characterizing the central core of the vortex is strong enough to cause a suction of the fluid from above. It (the backflow) is slightly shifted from the axis of the device indicating the three dimensional nature of the flow. Figures $7 \mathrm{a}$ and $7 \mathrm{~b}$ showing the computed contours of the radial and tangential components of the mean velocity using the RSM confirm that the axisymmetric flow assumption is certainly not justified.

\subsection{Velocity Fluctuations (rms)}

Figures 8 and 9 show radial profiles of the tangential and axial components of the fluctuating velocity (the rms values) respectively, at $\mathrm{z}=765 \mathrm{~mm}$ and $\mathrm{z}=855$ $\mathrm{mm}$ and at the angles $\theta=0,50^{\circ}, 110,180,230,290^{\circ}$. Under the exit tube, the turbulence is relatively intense 
in the central region where the maximum values of the axial and tangential velocity fluctuations are found. There, the relatively high straining between ascending and the descending streams is probably the main production mechanism of the turbulent energy. The turbulence is somewhat anisotropic: the axial Reynolds normal stress, produced essentially by the radial gradient of the axial mean velocity is slightly greater than the tangential Reynolds normal stress. In the boundary layer near the wall of the outer cylinder, an appreciable increase in the tangential component of the fluctuating velocity is noticed. Near the bottom of the cyclone, at $\mathrm{z}=855 \mathrm{~mm}$, the relatively intense turbulence in the core diminishes slightly. On the contrary, the weak turbulence, in the annular space away from the centre, undergoes a slight intensification.

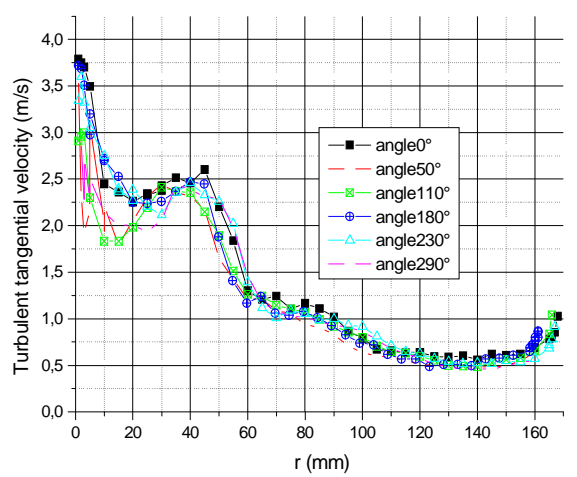

a) At $z=765 \mathrm{~mm}$

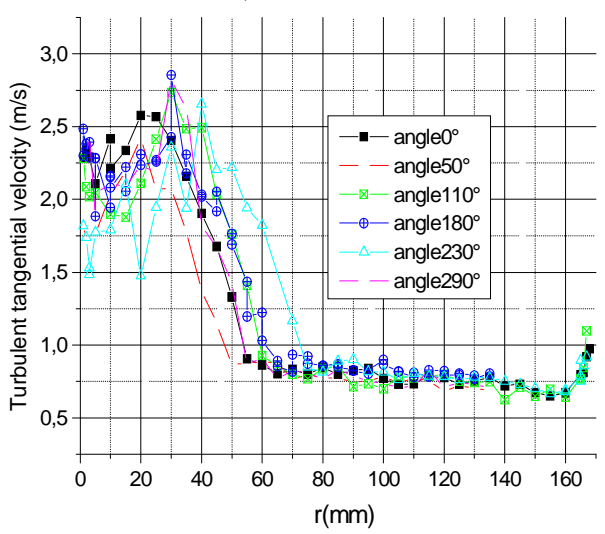

b) At $\mathrm{z}=855 \mathrm{~mm}$

Fig. 8. Profiles of tangential component of the fluctuating (rms) velocity.

\section{Conclusions}

An experimental study and a numerical simulation of the turbulent flow under the exit tube of a cyclone separator is presented. Radial profiles of the axial and tangential components of the mean and fluctuating velocities are obtained at different axial positions and angles in the cylindrical device. The RSM turns out to be a better turbulence model than the $\mathrm{k}-\varepsilon$ in predicting this kind of cyclonic flow. The results show the three dimensional nature of the flow; the existence, underneath the vortex finder, of a quasi-forced vortex in the core region surrounded by a quasi-free vortex in the annular space; an oscillation of the axis of the vortex with respect to the axis of the geometry.

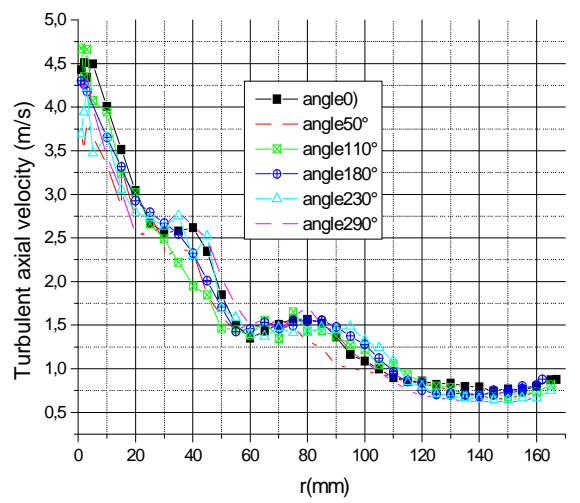

a) At $\mathrm{z}=765 \mathrm{~mm}$

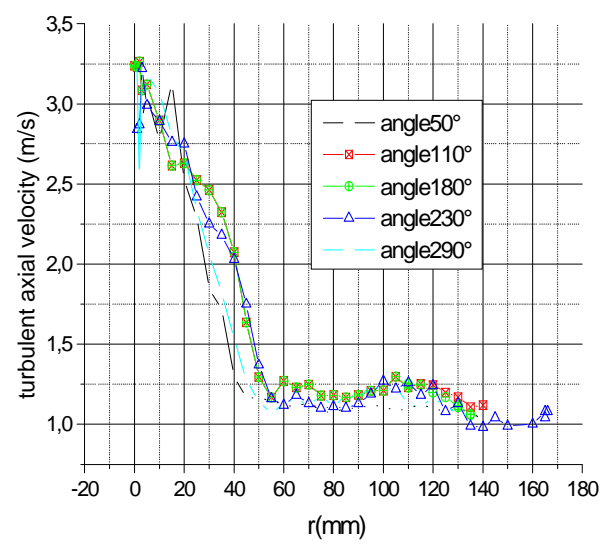

b) At $\mathrm{z}=855 \mathrm{~mm}$

Fig. 9. Profiles of the axial component of the fluctuating (rms) velocity.

\section{REFERENCES}

Ayers, W.H., F. Boysan, J. Swithenbank and B.C.R. Ewan (1983). Theoretical modelling of cyclone performance. Filtech conference.

Boysan, F., W.H. Ayer and J. Swithenbank (1982). A fundamental mathematical modelling approach to cyclone design. Trans. I.C.E. 60, 222-230.

Brennan, M.S. (2003, December). Multiphase CFD simulations of dense medium and classifying hydrocyclones. In Proceedings of the $3^{\text {rd }}$ International conference on CFD in Minerals and Process Industries, Melbourne, Australia.

Derksen, J.J. and H.E.A. Van den Akker (2002). Simulation of Vortex Core Precession in a Reverse-Flow Cyclone. AIChE Journal 46(7).

Frank, T., J. Schneider and E. Wassen, (1999, July). Experimental and Numerical Investigation of Particle Separation in a Double Cyclone Separation. In Proceedings of the $8^{\text {th }}$ Int. Symposium on gas-particles flow, ASME Fluids 
Engineering Division Summer Meeting, San Francisco, CA, USA.

Frank, T., E. Wassen and Q. Yu (1998, June). Lagrangian Prediction of Disperse Gas-Particle Flow in Cyclone Separators. In Proceedings of the $3^{\text {rd }}$ Int Conf. On Multiphase Flow, Lyon, France.

Fraser, S.M. and M.Z. Abdullah (1995). LDA Measurements on a Modified Cyclone. ASME Laser Anemometry, 229, 395-403.

Hishoda, H. (1996). Three-Dimensional Simulation of Air Cyclone and Particle Separation by a RevisedType Cyclone. Colloids and surfaces, Physicochemical and Engineering Aspects 109, 112.

Launder, B.E., G.J. Reece and W. Rodi (1975). Progress in the development of a Reynolds stress turbulence closure. J. Fluid Mech. 68, 537-566.

Lien, F.S. and M.A. Leschziner (1994). Assessment of Turbulent Transport Models Including Non-Linear RNG Eddy-Viscosity Formulation and SecondMoment Closure. Computers and Fluids 23(8), 983-1004.

Matvienko, O.V., J. Dück and T. Neesse (2001, March). Mathematical simulation of hydrocyclone hydrodynamics. In Proceedings of the International Congress for Particle Technology, Nürnberg.

Monredon, T.C., K.T. Hsien and R.K. Rajamani (1992). Fluid flow model of the hydrocyclone: An investigation of device dimensions. Int. J. of Mineral Processing 35, 65-83.

Obermair, S., J. Woisetschläger, G. Standiger (2001, October). The Flow in the Dust Outlet Geometry of Gas Cyclone and its Effects on Separation Efficiency. Filtech conference, Düsseldorf, Germany.
Ogawa, A. (1981, September). Analysis of loci of the fine solid particles and the flow pattern of air near the inlet pipe region in cyclone dust collectors. In Proceedings of International Symposium on Powder Technology 81, Kyoto, Japan, 609-616.

Peng, W., A.C. Hoffman, P. Boot, A. Udding, H.W.A. Dries and J.J. Kater (2001, March). Determining the Best Modelling Assumptions for Cyclones and Swirl Tubes by CFD and LDA. In Proceedings of the International Congress for Particle Technology, Nürnberg.

Potel, V., A. Donnot, M.O. Rigo (1997, September). Etude expérimentale de l'axisymétrie d'un écoulement cyclonique. In Proceedings of the $13^{\text {ème }}$ Congrès Français de Mécanique, Poitiers, 135-138.

Raoufi, A., M. Farzaneh, M. Shams and R. Ebrahimi (2007, July). Numerical optimization of cyclone vortex. In Proceedings of the 6th International Conference on Multiphase Flow, ICMF 2007, Leipzig, Germany.

Rodi, W. (1980). Turbulence Models and their application in hydraulics. International Association for Hydraulic Research.

Rotta, J.C. (1961). Turbulent boundary layers in incompressible flows. Progr. In Aeronaut. Sc. 2, Pergamon Press.

Slack, M.D., S. Del Porte and M.S. Engelman (2003). Designing automated computational dynamics modelling tools for hydrocyclone design. Fluent Europe Ltd, Sheffield.

Zhou, L. and X. Soo (1990). Gas solid flow and solid collection in a separator. Powder Technology 63, 45-53. 